linienförmigen Deckblättern besetzt, schlank, die obersten Aeste einfach; das Ganze bildet eine lockere weitläufige Rispe. Die Blätler flachgekerbt, ganz kahl oder nur oben kahl und unten steifhaarig; die grundständigen oder untern Stengelblätter, oft bis zu den Blüthenästen, vêrkehrte eirund, keilförmig in den Blattstiel verschmälert, an der Spitze stumpf, die obersten eiförmig, langzugespitzt, sitzend, denselben Stengel umfassend, nicht selten aber, ausser den untersten breitlanzettlich langzugespitzt, am Rande wellig, alle, bis auf die linienförmigen der Rispe, (die obersten nur wenig) gekerbt. Die Aeste tragen von oder über die Hälfte $\mathbf{3}-\mathbf{5}$ Blüthenstiele, die untern Blüthenstiele sind mit 1-a Deckblättern besetzt, lang, dünn, länger als die aufrechte Blume. Der Kelch kahl oder drüsig punktirt, die Zipfel pfriemlich, spilz, abstehend, 2-3mal länger als der Fruchtknoten, oft um die Hälfte kürzer als die Blumenkrone, oft von gleicher Länge, am Grunde mit einem oder mehreren kleinen, kaum bemerkbaren Zähnen. Die Blumenkrone nach dem Grunde schmäler zulaufend, bis zu 1/3 fünfspaltig, die Zipfel eirund-lanzetllich, spitz, auch um die Mittagszeit gerade. Der Griffel mit den Narben die Eirsschnitte der Krone erreichend. Die Kapsel verkehrt-eiförmig, an der Basis abgestutzt, 10nervig, zur Seite oben mit Löchern aufspringend.

Auf trockenen steinigen Bergwiesen an der Donau und bei den Herkules-Bädern im Banat, Mai, Juni.

Lugos, im Februar 1857.

\title{
Verzeichniss
}

derjenigen Pflanzen, die bisher in dem böhmischen Erzgebirge und in der Gegend von Rothenhaus und Umgebung aufgefunden worden sind.

Von A. Roth.

(Fortsetzung).

XXXVII. Gr a n a te en.

XXXVIII. O nagarie en.

Epilobium angustifolia L. Rothenhauser Waldrevier auf Holzschlägen Juli 1843 (A. Rot h).

" $\beta$. albiflorum. Am Wege von Rothenhaus nach Sadschitz Juli 1854. Rothenhauser Waldrevier beim Neustein 9. Aug. 1849 (A. R oth).

parviflorum Sch r eber. Sumpfwiesen bei Rothenhaus Juli 1845 (A. Roth).

montanum L. Brüxer Schlossberg 15. Juli 1851. Im Rothenhauser Park 23 Juli 1851 (A. R oth).

"var, albiflorum. Roihenhauser Park am Wegrande gegen den Fürstenbrunn Juli 1846 (A: R o th).

" $\gamma$. collinum. Bruxer Schlossberg 15. Jnli 1851. Rothenhaus am Ziegenberg 23. Juli 1852 (A. R oth).

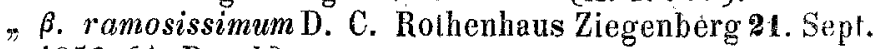
$1852(A, R$ oth). 
palustre L. An Wiesengräben bei Rothenhaus Aug. 1848 (A. Ro t h).

" $\beta$. hyssopifolium R c h b. Prossnitz 10. Juli 1851. Göllersdorfer Wiesen 1. Ang. 1851. An Wiesengräben bei Rothenhaus 12. Aug. 1851 (A. R oth).

tetragonum L. An Wassergräben bei Seehäusel nächst Kaaden Juli 1854 (Dr. $K$ n a f).

roseum $\mathrm{S} \mathrm{chreb}$ er. Bei Karlsbad ( $0 \mathrm{rtm} \mathrm{m} n)$. Rothenhaus in verlassenen Steinbrüchen Juli 1843 (A. Roth).

Oenothera biennis L. Im Rothenhauser Park Juli 1843 (A. Roth). Circaea intermedia $\mathrm{Eh} \mathrm{rh}$. Rothenhauser Waldrever Teltsch, an schattigen Orten in der Nähe der Brettmühle. Aug. 1844. Am Bache bei der Gabrielamühle in Rothenhaus 23. Aug. 1850 (A. Roth).

XXXIX. Ha lor a g e e n.

Myriophyllum verticillatum L. In Gräben der Kommerer Seewiesen 27. Juli 1850 (A. R o th).

$$
\text { XL. Hippurideen. }
$$

Hippuris vulgaris L. Auf Launer Wiesen, unter den Brückendurchlässen in stehendem Wasser 1. Juni 1844 (A. R o t h).

XLI. Callitrichieen.

Callitriche stagnalis $\mathbf{S}$ c o p. In Wassergräben der Kommerer Seewiesen 27. Juli 1850. Auf Sumpfwiesen am Dorfbach gegen den Seeberg bei Rothenhaus 29. Juli 1850 (A. R oth).

hamulata $\mathrm{K} \ddot{\mathrm{u}} \mathrm{z}$ ing. Rothenhaus im Dorfbach 11. Juli 1852 (A. Roth).

autumnalis $\mathrm{K} \ddot{\mathrm{t}} \mathrm{z}$. In der Bila bei Brüx. (A. Roth).

XLII. C er at op hylle en.

Ceratophyllum demersum L. In einem Teiche bei Kommern 6. Sept. 1854 (A. R o th).

XLIII. L y thrarieen.

Lythrum Salicaria L. Rothenhauser Park unter Gesträuch 23. Aug. $1851(A . R$ oth).

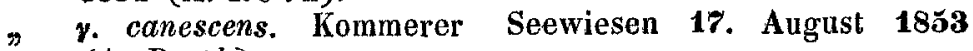
(A. Roth).

Peplis Portula L. Rothenhaus beim obern Röhrteich Juli 1842. Im neuen Fabriksteich bei der Pirkner Papiermühle 1. August 1843 (A. Roth).

XLIV. XLV, \& XLVI.

XLVII. Cucurbitaceen.

Bryonia alba L. Rothenhaus an Zäunen und im Park unter Gebiisch an vielen Orten 26. Juni 1841 (A. Roth). 
XLVIII. P ort a lace en.

Montia rivularis Gmel. Rothenhaus im Dorfbach in Wiesengräben 11. Juli $1852(A . R \circ \mathrm{th})$.

XLIX. P aron ychieen.

Herniaria glabra. L. Am Rothenhauser Ortsplatz und im Park 26. Juni 1842 (A. Roth.)

L. Scleran the en.

Scleranthus annuus L Auf sandigen Feldern bei Rothenhaus 26. Juni 1842 (A. R oth).

perennis L. Rothenhaus, Ziegenberg, 24. Juni (A. R $0 t h)$;

\section{Crassulace en.}

Sedum maximum Sut. Rothenhauser Waldrevier an Felsen August (A. Roth).

villosum L. Auf torfhaltigen Wiesen in Quinau Juli 1842. Auf Prossnitzer Wiesen 10. Juli 1851. (A. R ot h).

album L. Am Mileschaner Berg 9. Juli 1843. Brüxer Schlossberg 18. Juli 1850 (A. R o $t h)$.

acre L. Rothenhauser Park Juni 1842 (A. Roth).

sexangulare L. Rothenhauser Park Juli 1842 (A, Roth).

reflexum L. Rothenhauser Park und Waldrevier 5. August 1813

(A. Roth).

Sempervivum tectorum L. Auf Mauern in Czernowitz Aug̣nst 1843 (Dr. Kna f).

soboliferum $\mathrm{S}$ i m s. Auf Mauern in Rothenhaus Juli 1815 (A. R o th). LII. Cact e e n.

(Fortsetzung folgt).

\section{Correspondenz.}

- Goth a im April 1857. - Bezüglich der Collomia grandiflora $\mathrm{D}$ o $\mathrm{u} \mathrm{g}$ l. theile ich lhnen mit, dass dieser im nordwestlichen Amerika heimischen Polemoniacee unser deutsches Klima besonders zu behagen scheint, denn schon früher erschien sie bei uns in Gotha als Gartenflüchtling auf einem nahe bei der Stadt gelegenen Kalkberge, obwohl nur in einzelnen Excmplaren und bald wieder verschwindend, lediglich ein der Zucht des Gärtners entsprungener Fremdling. Seit etwa acht Jahren jedoch hat sich diese schöne Pflanze, ohne dass man weiss woher, an dem circa drei Stunden von hier entfernten Flüsschen Apfelstädt in der Nähe des Ortes gleichen Namens u n d von da a mehrere Stunden zu beiden Seiten des Ufers entlang und im Beete des im Sommer fast ganz versiechenden Flüsschens eingebürgert und ist eine nun völlig akklimatisirte Bürgerin der deutschen Flora geworden. Das genannte Flüsschen führt Unmassen von Kies rom Thüringer Walde herab und bildet in seinem Beete und an seinen Ufern Kiesbäder and Abschwemmungen, in welchem die schöne Collomia grandiflora mit ihren hellfeischfarbenen Blüthen in 